\title{
ELECTRONIC PROPERTIES AND APPEARENCE OF A MARTENSITIC TRANSFORMATION IN B2-TYPE COMPOUNDS (TiNiX WITH X = Fe, Ru, Os)
}

R. KUENTZLER and Y. DOSSMANN

I.P.C.M.S., Unité Mixte 380046, CNRS, 3 Rue de l'Université, F-67084 Strasbourg, France

\begin{abstract}
The electronic properties of pseudo-binary transition metal alloys of Bz-type structure TiNix ( $X=F e, R u, O s)$ are studied. The electronic specific heat coefficient $\gamma$ show systematically a peak, or a "plateau", at the concentration where a martensitic phase appears and corresponds to the boundary limit of the structural map.
\end{abstract}

1. Introduction.

The subject of martensitic phase transformation is one of the considerable scientific as well as technological interest. These metals have the following common characteristic features well established by $\mathrm{X}$-ray, electron, and neutron diffraction experiments /1/:

(1) The phonon dispersion curve shows TA branches with a dip at $q=q_{0}\left(q_{0} \neq 0\right)$, a wawe number characteristic at low-temperature phases which slightly deepens with decrease temperature $/ 2 /$. However, the frequencies of relevant phonon mode never approach zero even at the transition temperature.

(2) "Precursor" phenomena such as tweed patterns in electron micrograph /2/ and diffuse incommensurate spots in $X$-ray diffraction patterns have been observed well above the transition.

Very little is known on the electronic properties of these alloys (excepted far A15-type structure alloys in relation with superconductivity). Whe have undertaken a systematic study of pseudo-binary transitions metal alloys of B2-type structure using essentially ZrRu and TiNi as matrix. We consider here TiNiX, which is investigated with $X=M n, F e, C o, R u, O s, P d$ and focuse on TiNiFe. Low temperature specific heat results are presented. 


\section{Experimental results}

The pseudo-binary system $\mathrm{Ti}_{50}{ }^{\mathrm{Ni}}{ }_{50-x} \mathrm{Fe}_{x}$ is the most studied system showing a martensitic transformation since the substitution of $\mathrm{Fe}$ to $\mathrm{Ni}$ in TiNi at low concentration reduces substantially the martensitic transformation temperature $M_{s}$ but does not change appreciably the premartensitic temperature $T_{R}$ yielding good condition to study premartensitic effect. The sequence of transformations observed (on cooling) in the $\mathrm{Ti}_{50}{ }^{\mathrm{Ni}}{ }_{50-x} \mathrm{Fe}{ }_{x}$ alloys is as follow /2/: parent phase (B2) --> incommensurate phase (distorded cubic) $\rightarrow$ commensurate phase (rhombohedral) --> martensitic phase (monoclinic).

Whe have prepared samples by arc melting under argon atmosphere over the whole concentration range $0 \leq x \leq 50$. Annealing was performed, $950^{\circ} \mathrm{C} / 48 \mathrm{~h}$, and cooling obtained by furnace cutting and also by quenching for comparaison for a few concentrations. X-ray analysis was systematically realized to check the structure at room temperature but no variation with temperature has been done at this stage. The low temperature specific heat has been investigated in the temperature range $1.5-20 \mathrm{~K}$ (and up to $40 \mathrm{~K}$ for certain alloys) using a quasi-adiabatic method controlled by computer.

The variation of $M_{s}$ with increasing $x$ shows a decrease /3/ but for given $x$ certain discrepencies exist among the results reported by different authors. Heat treatments and the cooling method affects also $M_{s}$ value. For $x=5$ at $\%$ Fe, $M_{s}<77 \mathrm{~K}$ following Hwang /4/ as an example of value and $M_{s} \simeq 0$ for $x \simeq 7$ at $\% \mathrm{Fe}$.

As an example we plot in fig 1 , C/ $\mathrm{T}$ versus $\mathrm{T}^{2}$ for $\mathrm{Ti}_{50}{ }^{\mathrm{Ni}}{ }_{42} \mathrm{Fe}_{8}$, $\mathrm{Ti}_{50} \mathrm{Ni}_{42} \mathrm{Ru}_{8}$ and $\mathrm{Ti}_{50} \mathrm{Ni}_{45} \mathrm{Os}_{5}, \mathrm{Fe}, \mathrm{Ru}$ and $\mathrm{Os}$ being isoelectronic. It can be observed that at low temperature the variation $\mathrm{C} / \mathrm{T}$ versus $\mathrm{T}^{2}$ is linear $(\mathrm{C}=$ $\gamma \mathrm{T}+\beta \mathrm{T}^{3}$ ), but for increasing temperature an abnormal downturn appears. For low and high concentration alloys the regular upturn is observed due to the deviation from the Debye approximation for increasing temperature. For $\mathrm{X}=\mathrm{Fe}$ This regular behaviour is observed for $x\langle 5$ at $\%$ and $x\rangle 10$ at $\%$.

From the determination of the slope $\beta_{,} \theta_{D}$ is determined and plotted as a fonction of $\mathrm{x}$ for $\mathrm{Ti}_{50} \mathrm{Ni}_{50-x} \mathrm{Fe}_{x}$ in $\mathrm{fig} 2$.

A minimum value $(210 \mathrm{~K})$ is reached for $x \simeq 8$ at $\%$ but stays low up to $x=$ 12 at $\%$. The same behaviour is observed for $X=R u, O s$. The downturn in the plot $C / T$ versus $T^{2}$ and the low ${ }_{D}$ values for the concentrations in the vicinity of the concentration at which martensitic transformation appears ( $M_{s} \simeq 0$, can be attributed to phonons softening. The coefficient $\gamma_{\text {, }}$ proportional to the density of states at the Fermi level $n\left(E_{F}\right)$ is plotted versus $x$ in fig. 3 . 


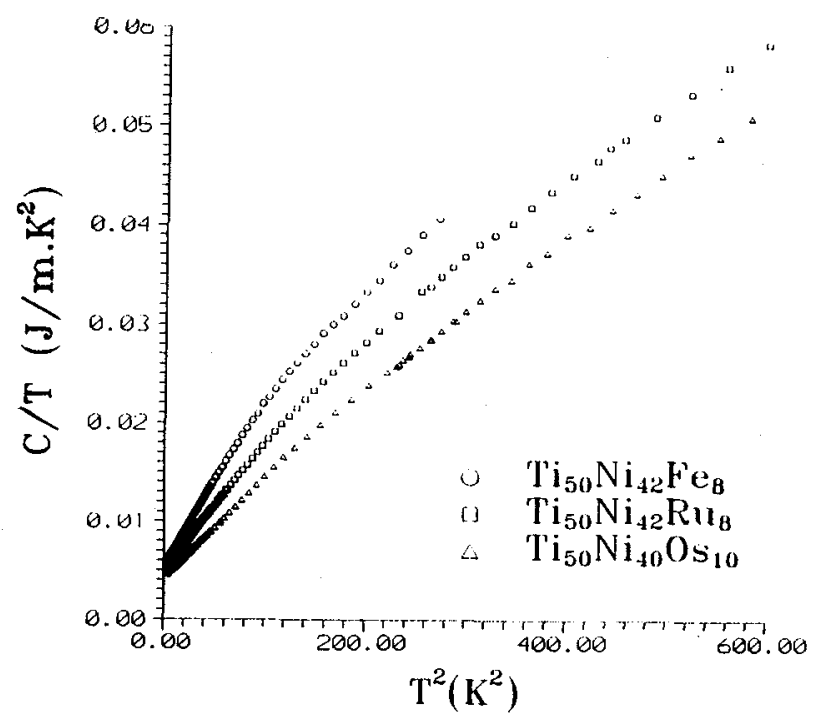

Fig 1 Low temperature specific heat of $\mathrm{Ti}_{50} \mathrm{Ni}_{42} \mathrm{Fe}_{8} \cdot \mathrm{Ti}_{50} \mathrm{Ni}_{42}{ }^{\mathrm{Ru}}{ }_{8}$ and $\mathrm{Ti}_{50}{ }^{\mathrm{Ni}}{ }_{45} \mathrm{OS}_{5}$, in the plot $\mathrm{C} / \mathrm{T}$ versus $\mathrm{T}^{2}$.

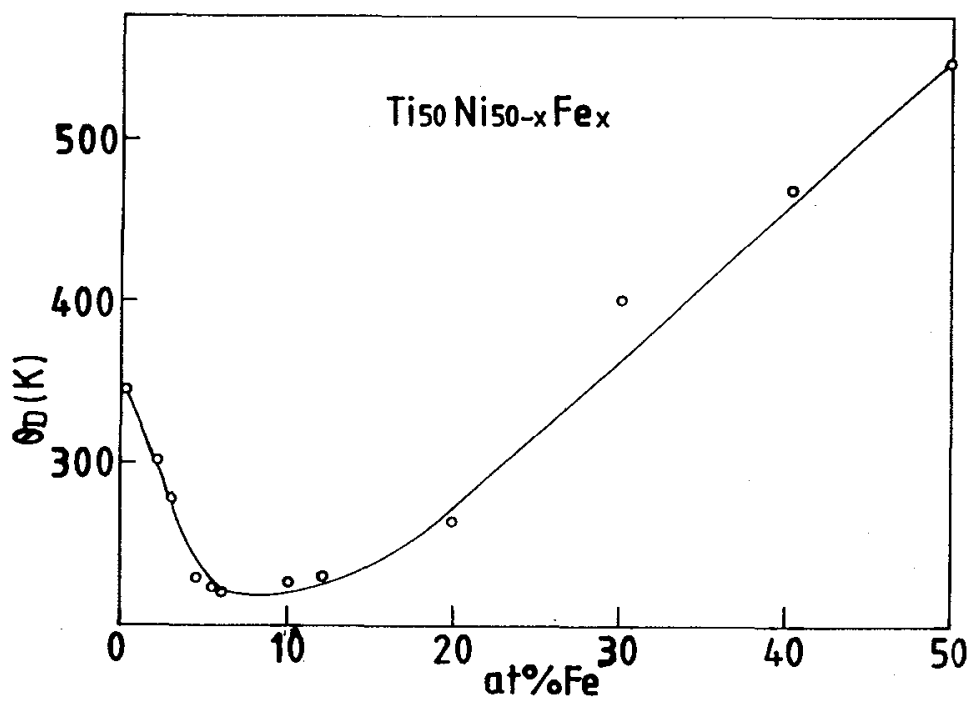

Fig 2 Debye temperature $\theta_{D}$ as a fonction of $\mathrm{x}$ of $\mathrm{Ti}_{50} \mathrm{Ni}_{50-x} \mathrm{Fe}$. 


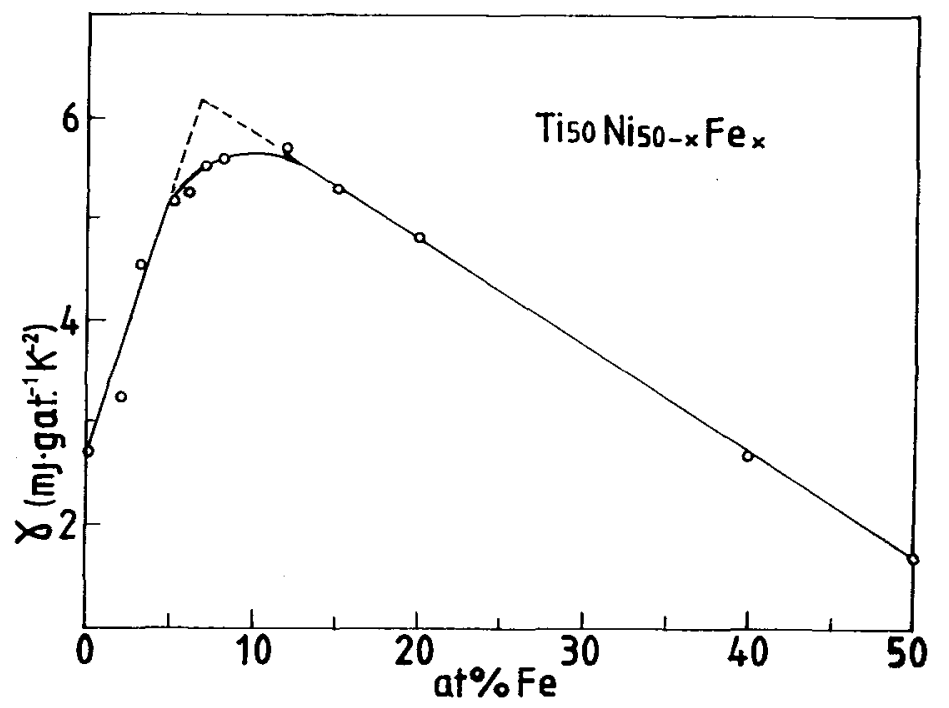

Fig 3 Electronic specific heat coefficient $\gamma$ as a fonction of $\mathrm{x}$ of $\mathrm{Ti}_{50} \mathrm{Ni}_{50-x} \mathrm{Fe}_{x}$.

A rounded peak can be observed in the vicinity of $x=8$ at $\&$ Fe where the martensitic transformation appears. The decrease is sharp for low $x$ values the martensitic phase side of the curve - and soft for large $x$ values - the parent phase B2 side of the curve. The same behaviour is observed for TiNiRu and TiNios isoelectronic systems but the rounded peak is larger and can be assimilated to a "plateau". The existence of a "plateau" instead of a peak in $\gamma(x)$ is puzzling. Is it due to metallurgical inhomogeneity with dentrites of B2 phase in the martensitic phase as it seems to be the case for ZrRuPd or does it corresponds to a "premartensitic" concentration range? This is not definitively established and more work should be done.

\section{Discussion}

Recently a theorical model for determining the stability of ordered structures (OS) based on electronic parameters has been developed $/ 5,6 /$. Structural maps, ( $\delta \mathrm{d}, \mathrm{Nd})$ where $\delta \mathrm{d} \cong \Delta \mathrm{Nd}$, yielding to the possible existence of ordered alloys for a given ordered structure-type were constructed, like B2-type. We have shown from experimental studies of low temperature specific heat that low $\gamma \simeq \mathrm{n}\left(E_{F}\right)$ values are observed for stable os $/ 7 \%$. We have suggested $/ 8 /$ that crossing the boundary limit of a given structural map at $T$ $=0 \mathrm{~K}$ ( B2-type structure) would correspond to ordered alloys showing at low temperature a martensitic transformation. With this picture in mind, high $\gamma$ values, being the signature of an os instability, should feature the crossing of the boundary limit with pseudo-binary transition metal alloys. We observed 
effectively high $\gamma$ values for $M_{s} \simeq 0$ in our systems when crossing the boundary limit of the structural map.

Theorical DOS calculations would be attractive for $B 2$ and AuCd-type structures to compare with the experimental results. We have the complete DOS calculation for TiNi for Bz-type structure showing a gap with $E_{F}$ nearly inside of it, which is coherent with expe-rimental results. For the low temperature B19'-phase, the high peak of DOS (in the non occupied band) disappears and $n\left(E_{F}\right)$ decreases but the other features are maintained /9/. Microcopic description of the diffusionless phase transformations are highly desired to go further in the interpretation but there is still much controverse about. Recently however / 10-12/, significant progress in under-standing of the nature of martensitic transformations has been made, in which all of the ideas are more or less based on the lattice instability against $\mid 110\}\langle 110\rangle$ shear strains. Anharmonicity models are proposed instead of soft-mode mechanisms based on charge density waves or Fermi surface effects put forth by many authors and this leaves open further discussion on our work under progress.

\section{References}

/1/ SHAPIRO M., NODA Y., FUJII Y., YAMADA Y.

Phys. Rev. B 30 (1984) 4314

$12 /$ HWANG C.M., MEICHLE M., SALAMON M.B., WAYMAN C.M.

Phil. Mag. A 47 (1983) 9

13/ Shabaloveskaya S.A. Phys. Stat. Sol. (b), 132 (1985) 327

/4/ HWANG C.M., WAYMAN C.M., Scripta Met. 17 (1983) 1345

/5/ BIEBER A., GAUTIER F., Acta Met. 34 (1986) 2291

/6/ TURCHI P.E.A., Mat. Sci. Engn. A 127 (1990) 145

/7/ KUENTZLER R., WATERSTRAT R.M., TUREK P., BIEBER A., Proc. Int. Conf. on Phase Transformations in solids, (edited by T. Tsakalakos) North-Holland, New York (1984) 393

18/ KUENTZleR R., WATERstrat R.M., J. Less Comm. Met. 125 (1986) 261

19/ KULKova S.E., EGORUSHKIN V.E., KALCHIKHIN, Solid state Comm. 77 (1991) 667.

/10/ KrumhansL J.A., YAMAdA Y., Mat. Sci. Engn. A 127 (1990) 167 and references therein.

/11/ LINGARD P.A., MOURITSEN 0.G. Phys. Rev. B 41 (1990) 688 /12/ GUENIN G., ClAPP P.C.,Proc. Int. Conf on Martensitic Transformations, ICOMAT-(1986), ( Jap. Inst. Met. Sendai, 1987) 171 